EPJ manuscript No.

(will be inserted by the editor)

\title{
The Goldberger-Miyazawa-Oehme sum rule revisited
}

\author{
V.V. Abaev ${ }^{1}$, P. Metsä2 ${ }^{2}$ and M.E. Sainio ${ }^{3}$ \\ 1 Petersburg Nuclear Physics Institute, Gatchina 188300, Russia \\ 2 Department of Physical Sciences, P.O. Box 64, 00014 University of Helsinki, Finland \\ 3 Helsinki Institute of Physics, P.O. Box 64, 00014 University of Helsinki, Finland \\ Received: date / Revised version: date
}

\begin{abstract}
The Goldberger-Miyazawa-Oehme sum rule is used to extract the pion-nucleon coupling constant from experimental $\pi \mathrm{N}$ information. Chiral perturbation theory is exploited in relating the pionic hydrogen $s$-wave level shift and width results to the appropriate scattering lengths. The deduced value for the coupling is $f^{2}=0.075 \pm 0.002$, where the largest source of uncertainty is the determination of the $s$-wave $\pi^{-} p$ scattering length from the atomic level shift measurement.
\end{abstract}

PACS. 13.75.Gx Pion-baryon interactions - 14.20.Dh Protons and neutrons

\section{Introduction}

The Goldberger-Miyazawa-Oehme [1] sum rule relates the coupling constant of the pion-nucleon interaction, $f^{2}$, to the difference of the $\pi^{-} p$ and $\pi^{+} p s$-wave scattering lengths and to the weighted integral of the difference of the $\pi^{-} p$ and $\pi^{+} p$ total cross sections. By evaluating the forward dispersion relations at the physical threshold, $\omega=\mu$, we obtain [2]

$$
(1+\mu / m)\left(a_{\pi^{-} p}-a_{\pi^{+} p}\right) / \mu=4 f^{2} /\left(\mu^{2}-\omega_{B}^{2}\right)+2 J^{-},(1)
$$

where $\mu$ is the (charged) pion mass, $m$ is the proton mass, $\omega_{B}=-\mu^{2} / 2 m$ and

$$
J^{-}=1 /\left(4 \pi^{2}\right) \int_{0}^{\infty}\left(\sigma_{\pi^{-} p}^{\operatorname{Tot}}-\sigma_{\pi^{+} p}^{\text {Tot }}\right) / \omega d k .
$$

The $s$-wave $\pi^{-} p$ and $\pi^{+} p$ scattering lengths are denoted by $a_{\pi^{-} p}$ and $a_{\pi^{+} p}$ respectively and $\omega=\sqrt{\mu^{2}+k^{2}}$, where $k$ is the pion laboratory momentum.

It is well known that eq. (1) is not a precise means to determine the value of the pion-nucleon coupling $f^{2}$, but it is the natural first step in the process of extracting the value from the pion-nucleon data. Furthermore, the sum rule makes the connection between the uncertainties in the data and in the coupling constant more transparent.

It is the aim of the present paper to examine the ingredients of eq. (1), two scattering lengths and integral $J^{-}$, and determine the pion-nucleon coupling strength $f^{2}$. The implications of the pionic hydrogen measurements to the sum rule are discussed in sect. 2 , the $s$-wave $\pi^{+} p$ scattering length is addressed in sect. 3 and the integral $J^{-}$in sect. 4. Section 5 combines the information to extract a value for $f^{2}$ and in sect. 6 the conclusions are drawn.

\section{Pionic hydrogen}

The accurate measurements of the properties of pionic hydrogen at PSI provide a source of information on the pionnucleon amplitude very close to the physical threshold. The strong interaction level shift $\epsilon_{1 s}$, determined through the difference of the electromagnetic and measured transition energy difference

$$
\epsilon_{1 s}=E_{3 p-1 s}^{\mathrm{em}}-E_{3 p-1 s}^{\mathrm{meas}},
$$

has been measured to a precision of about $0.2 \%$ [

$$
\epsilon_{1 s}=-7.120 \pm 0.008 \pm 0.009 \mathrm{eV}
$$

The first error is due to statistics and the second due to systematics. There is a considerable improvement in accuracy compared with the earlier result [4]

$$
\epsilon_{1 s}=-7.108 \pm 0.013 \pm 0.034 \mathrm{eV}
$$

mainly because the molecular effects are better under control. However, the improvement is not reflected in the extraction of the $\pi^{-} p s$-wave scattering length. The connection between the level shift and the scattering lengths is provided by the formula [5]

$$
\epsilon_{1 s}=-2 \alpha^{3} \mu_{c}^{2}\left(a_{0+}^{+}+a_{0+}^{-}\right)\left(1+\delta_{\epsilon}\right),
$$

where $\mu_{c}$ is the reduced mass of the $\pi^{-} p$ system, $\alpha \simeq$ $1 / 137.036$ is the fine structure constant and $a_{0+}^{+}, a_{0+}^{-}$are the isoscalar and isovector $s$-wave $\pi \mathrm{N}$ scattering lengths respectively. The quantity $\delta_{\epsilon}$ evaluated next-to-leading order in isospin breaking and in the low-energy expansion has the value 5 ]

$$
\delta_{\epsilon}=(-7.2 \pm 2.9) \times 10^{-2} .
$$


The uncertainty of the $\pi^{-} p$ scattering length determined with eq. (6) is then dominated by the uncertainty in $\delta_{\epsilon}$, which in turn is dominated by the largely unknown lowenergy constant $f_{1}[5$. Potential models produce typically smaller correction factors $\delta_{\epsilon}$ with considerably smaller uncertainties [6,7]. However, here we rely on the chiral perturbation theory result [5] for $\delta_{\epsilon}$ and consequently the measured level shift of eq. (4) gives

$$
a_{\pi^{-} p}=0.0933 \pm 0.00291 / \mu,
$$

if errors in eq. (4) are added linearly. Here the identification $a_{\pi^{-}}=a_{0+}^{+}+a_{0+}^{-}$has been made.

The Deser-type formula [8] relating the isovector $s$ wave scattering length $a_{0+}^{-}$to the level width is [6]

$$
\Gamma_{1 s}=8 \alpha^{3} \mu_{c}^{2} q_{0}(1+1 / P)\left[a_{0+}^{-}\left(1+\delta_{\Gamma}\right)\right]^{2},
$$

where $q_{0}$ is the centre-of-mass momentum of the $\pi^{0}$ in the charge exchange reaction and $P=1.546 \pm 0.009$ is the Panofsky ratio [9], i.e. the ratio of the cross sections $\sigma\left(\pi^{-} p \rightarrow \pi^{0} n\right) / \sigma\left(\pi^{-} p \rightarrow \gamma n\right)$ at the threshold. The correction factor $\delta_{\Gamma}$ has been evaluated in leading order in chiral perturbation theory with the result [10]

$$
\delta_{\Gamma}=(0.6 \pm 0.2) \times 10^{-2}
$$

A potential model calculation [6] would give a different sign, but this correction factor is rather small in any case. The factor $\delta_{\Gamma}$ depends on the low-energy constant $f_{2}$ and, therefore, the uncertainty is smaller than for the factor $\delta_{\epsilon}$, which depends on the constant $f_{1}$.

The $s$-wave isovector scattering length can be solved from eq. (9)

$$
a_{0+}^{-}=\frac{0.08933}{1+\delta_{\Gamma}} \sqrt{\frac{\Gamma_{1 s}}{0.868 \mathrm{eV}}} 1 / \mu .
$$

The measured result for the width [4] $\Gamma_{1 s}=0.868 \pm$ $0.040 \pm 0.038 \mathrm{eV}$ then gives for the scattering length $a_{0+}^{-}=0.0888 \pm 0.00401 / \mu$. The relatively large error bar is largely due to the uncertainty in estimating the Doppler broadening. The new preliminary result for the width [11] with improvement in this respect, $\Gamma_{1 s}=0.823 \pm 0.019 \mathrm{eV}$, would give $a_{0+}^{-}=0.0865 \pm 0.00101 / \mu$.

\section{Discrete phase shift analysis for $\pi^{+} p$}

For the $\pi^{+} p$ interaction a discrete phase shift analysis has been performed in the range $k=0.077-0.725 \mathrm{GeV} / \mathrm{c}$ at 77 different momenta 12 . Tromborg corrections 13 have been used to extract the hadronic amplitudes from the experimental data. Forward scattering constraints [14] have been applied iteratively in the analysis. Additional constraints from total inelastic cross sections were used for partial wave inelasticities. Special care has been taken to incorporate in the analysis the experimental resolutions and acceptances. In this way only a small number of points had to be eliminated due to inappropriate angular dependence. The normalizations were allowed to float

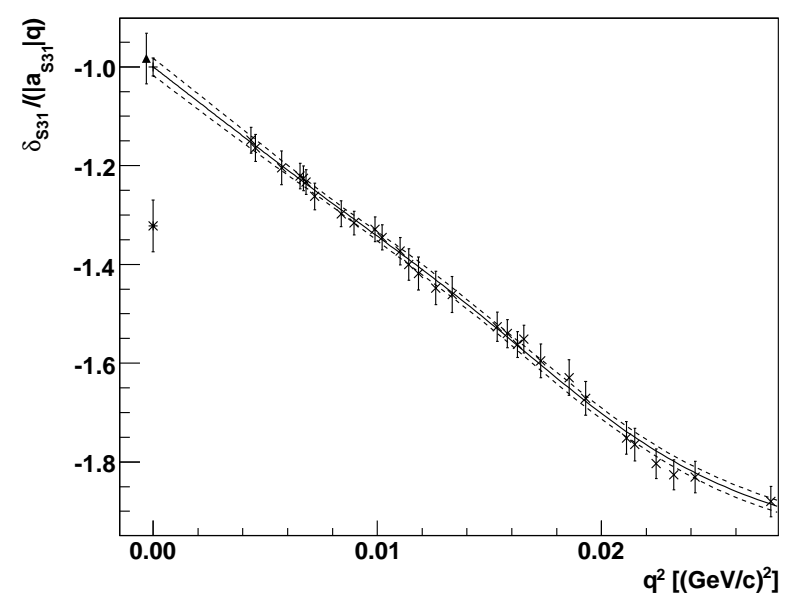

Fig. 1. The $\pi^{+} p s$-wave phase shift $\delta_{S 31}$ normalized with $\left|a_{S 31}\right| q$. The asterix denotes the KH80 value. The result of Matsinos et al. is shown by the triangle.

according to experimental information on the systematic uncertainty. Furthermore, the behaviour of the zero trajectories for transversity amplitudes was monitored to keep a smooth variation in the shape of observables and the extrapolation to the physical threshold was stabilized in this manner. Figure 1 shows the phase shift $\delta_{S 31}$ close to the threshold normalized with the product of the absolute value of the $s$-wave scattering length $a_{S 31}$ and the centreof-mass momentum $q$. The result can be parametrized with analytical forms in three different $q^{2}$ ranges such that in the lowest energy range the effective range approximation is used. The two dividing momenta have been taken as free parameters. It turns out that a two-parameter effective range approximation is valid up to $q \simeq 0.14 \mathrm{GeV} / \mathrm{c}$. The result for the scattering length $a_{S 31}$

$$
a_{S 31} \equiv a_{\pi^{+} p}=-0.0764 \pm 0.00141 / \mu
$$

is very stable. This result completes the discussion of the left hand side of eq. (1). The result can be compared with the recent figure of Matsinos et al. 15] $a_{\pi^{+} p}=-0.0751 \pm$ $0.00391 / \mu$ based on a fit with a low-energy model and with the FA02 solution of the GWU/VPI group [16 $a_{\pi^{+} p}=$ $-0.0911 \pm 0.00141 / \mu$. For the KH80 solution [17] of the Karlsruhe group $a_{\pi^{+} p}=-0.1010 \pm 0.00401 / \mu$.

\section{Integral $J^{-}$}

The integral $J^{-}$contains input from a number of sources. Results from total cross section measurements are available in the range $0.16-640 \mathrm{GeV} / \mathrm{c}$ for the $\pi^{-} p$ scattering and $0.16-340 \mathrm{GeV} / \mathrm{c}$ for the $\pi^{+} p$ scattering [18. These data have been corrected for the electromagnetic effects up to $k=0.725 \mathrm{GeV} / \mathrm{c}$ using the formalism of Tromborg [13. The corrections have been published only up to 0.655 $\mathrm{GeV} / \mathrm{c}$, but here we employ a smooth extrapolation up to $0.725 \mathrm{GeV} / \mathrm{c}$. The question of electromagnetic corrections 
Table 1. Contributions to $J^{-}(\mathrm{mb})$ of the different highenergy ranges of the laboratory momentum $k$.

\begin{tabular}{lll}
\hline Input & $10-350 \mathrm{GeV} / \mathrm{c}$ & $350-\mathrm{GeV} / \mathrm{c}$ \\
\hline Höhler [22] & 0.08786 & 0.01787 \\
Donnachie-Landshoff [23] & 0.09968 & 0.02514 \\
Gauron-Nicolescu 24] & 0.10665 & 0.02012 \\
PDG 25] & 0.09587 & - \\
Present work & 0.09609 & - \\
\hline
\end{tabular}

above the Tromborg range has been discussed in a number of articles 19,20. Here we do not, however, correct the data above the limit $0.725 \mathrm{GeV} / \mathrm{c}$, but we estimate the corresponding uncertainty in the value of $J^{-}$by making use of the prescription in 21. A smooth curve through the experimental points is drawn using the expansion technique [22], which guarantees that forward dispersion relations are satisfied. Details can be found elsewhere [14.

The integral $J^{-}$converges relatively slowly and the high-energy behaviour of the cross sections $\sigma_{\pi^{ \pm}}^{\text {Tot }}$ plays a significant role. Here we consider three different Regge type parametrizations for the asymptotic forms, i.e. forms which give estimates of the total cross sections beyond 350 $\mathrm{GeV} / \mathrm{c}$ : the parametrizations of Höhler [22], Donnachie and Landshoff [23] and Gauron and Nicolescu 24]. In the range $10-350 \mathrm{GeV} / \mathrm{c}$ the recent parametrization of the Particle Data Group [25] has been employed in addition to the Regge forms quoted above and the result from the fit with the expansion technique [14. The results of these parametrizations have been summarized in table 1 . The displayed number of significant figures is there only to help in the addition of the contributions of the different momentum ranges. The momentum range from the threshold to $10 \mathrm{GeV} / \mathrm{c}$ is split into two at $2.03 \mathrm{GeV} / \mathrm{c}$. For the low-energy section from the threshold to $2.03 \mathrm{GeV} / \mathrm{c}$ the experiment covers only part of the range. From the discussion in sects. 2 and 3 we can, by invoking isospin invariance, deduce values for the $\pi^{ \pm} p$ total cross sections at the physical threshold with the results $\sigma_{\pi^{-} p}^{\text {Tot }}=5.80 \pm 0.27 \mathrm{mb}$ and $\sigma_{\pi^{+} p}^{\text {Tot }}=1.47 \pm 0.05 \mathrm{mb}$. In the range from 0.08 to 0.16 $\mathrm{GeV} / \mathrm{c}$ there is information on the angular distributions, but no modern total cross section data. For this range we have performed a phase shift analysis with elastic $\pi^{ \pm} p$ input and fixed- $t$ constraints. Also, results from the discrete phase shift analysis for $\pi^{+} p$ discussed in sect. 3 have been used as cross checks. The remaining piece from the threshold to $0.08 \mathrm{GeV} / \mathrm{c}$ has been covered by a smooth interpolation. The contributions to integral $J^{-}$can then be evaluated with the results displayed in table 2 . There, in addition, results from the phase shift analyses of the Karlsruhe group and the GWU-VPI group are shown. A detailed discussion of the contributions of different momentum ranges can be found, e.g., in ref. [21].

Combining the values from different momentum slices yields the value for $J^{-}$given in table 3 . Also, results from some earlier evaluations have been displayed there. To as-
Table 2. Contributions to $J^{-}(\mathrm{mb})$ of the low and intermediate energy ranges of the laboratory momentum $k$.

\begin{tabular}{lll}
\hline Input & $0-2.03 \mathrm{GeV} / \mathrm{c}$ & $2.03-10 \mathrm{GeV} / \mathrm{c}$ \\
\hline KH80 [17] & -1.27853 & 0.10691 \\
KA84 [26] & -1.31266 & 0.13802 \\
FA02 [16] & -1.30213 & - \\
Present work & -1.29757 & 0.12046 \\
\hline
\end{tabular}

Table 3. The values for the integral $J^{-}(\mathrm{mb})$.

\begin{tabular}{ll}
\hline Source & $J^{-}(\mathrm{mb})$ \\
\hline Höhler-Kaiser [29] & -1.06 \\
Koch [27] & $-1.077 \pm 0.047$ \\
Gibbs et al. [28] & $-1.051 \pm 0.005^{\mathrm{a}}$ \\
Ericson et al. 21] & $-1.083 \pm 0.032$ \\
Present work & $-1.060 \pm 0.030$ \\
\hline a Statistical error only.
\end{tabular}

sign an error bar to our result for $J^{-}$, we note that the statistical uncertainty is quite small, about $0.007 \mathrm{mb}$. It is, however, hard to estimate the systematic uncertainty. The systematic normalization uncertainties of the data have been taken into account in the fitting procedure [14. Also, there remain discrepancies in the data, see the discussion, e.g., in ref. [29]. The $\Delta$-resonance gives a major contribution to the $J^{-}$-integral and in that range the two accurate experiments, Carter et al. [30] and Pedroni et al. [31, differ slightly, but in a systematic manner. The impact of the difference on the $J^{-}$is $0.012 \mathrm{mb}$. We adopt this as the number reflecting experimental systematic effects. Furthermore, the forward dispersion relations, which have been imposed as contraints, do need a value for the pion-nucleon coupling constant as input. Here the pionnucleon coupling constant has been allowed to vary and the effect on the error bar for the $J^{-}$integral is $0.001 \mathrm{mb}$. For the asymptotic part, where data do not exist, the average of the numbers in table 1 has been taken and the corresponding error is chosen such that all the displayed values fall within the errors. This gives as the estimate for the error $0.004 \mathrm{mb}$. If in the integrand for $J^{-}$involving $\sigma_{\pi^{-} p}^{\text {Tot }}-\sigma_{\pi^{+} p}^{\text {Tot }}$ a Coulomb correction of the type [21] is adopted in the range $0.725-2.03 \mathrm{GeV} / \mathrm{c}$, the value for $J^{-}$ changes by $0.6 \%$. Adding up all these errors, we obtain the quoted uncertainty $0.030 \mathrm{mb}$.

\section{Results and discussion}

From eq. (1) the pion-nucleon coupling constant can be extracted with the result

$$
\begin{aligned}
f^{2}= & \frac{1}{2}\left[1-\left(\frac{\mu}{2 m}\right)^{2}\right] \times \\
& {\left[\frac{1}{2}\left(1+\frac{\mu}{m}\right)\left(a_{\pi^{-} p}-a_{\pi^{+} p}\right) \mu-J^{-} \mu^{2}\right] . }
\end{aligned}
$$


The contribution to $f^{2}$ from the term involving the difference of the $\pi^{-} p$ and $\pi^{+} p$ scattering lengths is about $2 / 3$ and the $J^{-}$piece about $1 / 3$. With the scattering lengths and the integral $J^{-}$evaluated in sects. 2-4, the coupling constant becomes

$$
f^{2}=0.075 \pm 0.002
$$

This precision can not compete with the expected accuracy from other methods involving the dispersion relations for the $B$-amplitudes, but we shall use this range of possible values as input for the phase shift analysis with fixed- $t$ constraints.

In the discussion above isospin symmetry has been used only in fixing the threshold values for the total cross sections, a statement valid to $\mathcal{O}\left(p^{3}\right)$ in chiral perturbation theory. By invoking the isospin invariance we can relate $a_{\pi^{-} p}-a_{\pi^{+} p}=2 a_{0+}^{-}$, where the isovector $s$-wave scattering length is accessible through the atomic width measurement, eq. (11). The numbers for $a_{0+}^{-}$quoted in sect. 2, yield the coupling strength in the range $f^{2}=0.076-0.077$, i.e. within the error range quoted above. By making use of isospin invariance one can avoid the question of the consistency of the approach when one is extracting the hadronic $\pi^{-} p s$-wave scattering length in chiral perturbation theory and the $\pi^{+} p$ scattering length in a discrete phase shift analysis exploiting the Tromborg approach for the electromagnetic corrections. We are aware of one low-energy $\pi \mathrm{N}$ analysis with a complete treatment of both strong and electromagnetic effects to third order in chiral perturbation theory, the work of Fettes and Meißner [32. There, indeed, it is found that nonlinear pion-nucleon-photon coupling terms generate sizeable effects. However, the comparison in ref. 32. for the low-energy $\pi^{+} p s$-wave scattering with the Karlsruhe results, which also use Tromborg corrections, shows that the effect is relatively small there.

The potential model 6 corrections $\delta_{\epsilon}=(-2.1 \pm 0.5) \times$ $10^{-2}$ and $\delta_{\Gamma}=(-1.3 \pm 0.5) \times 10^{-2}$ would lead to coupling values in the range $f^{2}=0.077-0.078$ in agreement with ref. [21.

\section{Conclusions}

The GMO sum rule provides a tool to relate experimental information on pion-nucleon scattering from different sources. Also, it is relatively straightforward to analyze the error propagation. With the input from pionic hydrogen and a discrete phase shift analysis for the $\pi^{+} p$ interaction we obtain for the pion-nucleon coupling constant the value $f^{2}=0.075 \pm 0.002$. It turns out, that the largest uncertainty in determining a value for the pion-nucleon coupling constant is currently due to the large uncertainty in the correction factor $\delta_{\epsilon}$ for extracting the the $\pi^{-} p s$-wave scattering length from the $\epsilon_{1 s}$ hadronic level shift measurement. Assuming isospin invariance we can make use of the isovector $s$-wave scattering length $a_{0+}^{-}$which will be determined within $1 \%$ by the PSI group in the future.
We wish to thank A.M. Green for useful comments on the manuscript. One of us (PM) thanks the Magnus Ehrnrooth Foundation and the Waldemar von Frenckell Foundation for financial support. Support from the Academy of Finland and the Russian Academy of Sciences exchange grant is acknowledged. This work was supported in part by the EU Contract MRTN-CT-2006-035482, FLAVIAnet.

\section{References}

1. M.L. Goldberger, H. Miyazawa, R. Oehme, Phys. Rev. 99, 986 (1955).

2. J. Hamilton, W.S. Woolcock, Rev. Mod. Phys. 35, 737 (1963)

3. L.M. Simons, Int. J. Mod. Phys. A 20, 1644 (2005).

4. H.-Ch. Schröder et al., Eur. Phys. J. C 21, 473 (2001).

5. J. Gasser, M.A. Ivanov, E. Lipartia, M. Mojžiš, A. Rusetsky, Eur. Phys. J. C 26, 13 (2002) arXiv:hep-ph/0206068.

6. D. Sigg, A. Badertscher, P.F.A. Goudsmit, H.J. Leisi, G.C. Oades, Nucl. Phys. A 609, 310 (1996).

7. T.E.O. Ericson, B. Loiseau, S. Wycech, Phys. Lett. B 594, 76 (2004) arXiv:hep-ph/0310134.

8. S. Deser, M.L. Goldberger, K. Baumann, W. Thirring, Phys. Rev. 96, 774 (1954).

9. J. Spuller et al., Phys. Lett. B 67, 479 (1977).

10. P. Zemp, Ph.D. thesis, University of Berne (2004).

11. L.M. Simons, in arXiv:hep-ph/0610201, p. 8.

12. V.V. Abaev et al., work in progress.

13. B. Tromborg, S. Waldenstrøm, I. Øverbø, Phys. Rev. D 15, 725 (1977).

14. P. Metsä, HIP-2007-20/TH, 2007, unpublished.

15. E. Matsinos, W.S. Woolcock, G.C. Oades, G. Rasche, A. Gashi, Nucl. Phys. A 778, 95 (2006) arXiv:hep-ph/0607080.

16. R.A. Arndt, W.J. Briscoe, I.I. Strakovsky, R.L. Workman, M.M. Pavan, Phys. Rev. C 69, 035213 (2004) arXiv:nucl-th/0311089.

17. R. Koch, E. Pietarinen, Nucl. Phys. A 336, 331 (1980).

18. http://pdg.lbl.gov/2006/hadronic-xsections/hadron.html

19. J. Rix, R.M. Thaler, Phys. Rev. 152, 1357 (1966).

20. D.V. Bugg, A.A. Carter, Phys. Lett. B 48, 67 (1974).

21. T.E.O. Ericson, B. Loiseau, A.W. Thomas, Phys. Rev. C 66, 014005 (2002) arXiv:hep-ph/0009312.

22. G. Höhler, Pion-Nucleon Scattering, Landolt-Börnstein, Vol. I/9b2 (Springer, Berlin, 1983).

23. A. Donnachie, P.V. Landshoff, Phys. Lett. B 296, 227 (1992) arXiv:hep-ph/9209205.

24. P. Gauron, B. Nicolescu, Phys. Lett. B 486, 71 (2000) arXiv:hep-ph/0004066.

25. W.-M. Yao et al., J. Phys. G 33, 1 (2006).

26. R. Koch, Z. Phys. C29, 597 (1985).

27. R. Koch, Karlsruhe TKP 85-5, 1985, unpublished.

28. W.R. Gibbs, Li Ai, W.B. Kaufmann, Phys. Rev. C 57, 784 (1998) arXiv:nucl-th/9704058.

29. G. Höhler, F. Kaiser, Karlsruhe report, KfK 3027, 1980, unpublished.

30. A.A. Carter, J.R. Williams, D.V. Bugg, P.J. Bussey, D.R. Dance, Nucl. Phys. B 26, 445 (1971).

31. E. Pedroni et al., Nucl. Phys. A 300, 321 (1978).

32. N. Fettes, U.-G. Meißner, Nucl. Phys. A 693, 693 (2001) arXiv:hep-ph/0101030. 